\title{
How Learning to Read Changes the Cortical Networks for Vision and Language
}

Stanislas Dehaene, ${ }^{1,2,3,4} *$ Felipe Pegado, ${ }^{1,2,3}$ Lucia W. Braga, ${ }^{5}$ Paulo Ventura, ${ }^{6}$ Gilberto Nunes Filho, ${ }^{5}$ Antoinette Jobert, ${ }^{1,2,3}$ Ghislaine Dehaene-Lambertz, ${ }^{1,2,3}$ Régine Kolinsky, ${ }^{7,8}$ José Morais, ${ }^{7}$ Laurent Cohen ${ }^{9,10,11}$

${ }^{1}$ INSERM, Cognitive Neuroimaging Unit, Gif sur Yvette, 91191 France. ${ }^{2}$ CEA, DSV, I2BM, Neurospin center, Gif sur Yvette, 91191 France. ${ }^{3}$ University Paris-Sud 11, 91405 Orsay, France. ${ }^{4}$ Collège de France, 11 Place Marcelin Berthelot, 75005 Paris, France. ${ }^{5}$ SARAH Network-International Center for Neurosciences and Rehabilitation, QL 13, Lago Norte, 71.535-005 Brasilia, DF Brazil. ${ }^{6}$ Faculty of Psychology, University of Lisbon, 1600-214 Lisbon, Portugal. ${ }^{7}$ Faculty of Psychology, Université Libre de Bruxelles (U.L.B.), 1050 Brussels, Belgium. ${ }^{8}$ Fonds de la Recherche Scientifique (F.N.R.S), 1000 Brussels, Belgium. ${ }^{9}$ Université Pierre et Marie Curie-Paris 6, Faculté de Médecine Pitié-Salpêtrière, 75013 Paris, France. ${ }^{10}$ AP-HP, Groupe hospitalier Pitié-Salpêtrière, Department of Neurology, 75651 Paris, France. ${ }^{11}$ INSERM, CRICM, UMRS 975, Paris, France.

*To whom correspondence should be addressed. E-mail: stanislas.dehaene@gmail.com

Does literacy improve brain function? Does it also entail losses? Using functional magnetic resonance imaging, we measured brain responses to spoken and written language, visual faces, houses, tools and checkers in adults of variable literacy (10 were illiterate, 22 became literate as adults, and 31 were literate in childhood). As literacy enhanced the left fusiform activation evoked by writing, it induced a small competition with faces at this location, but also broadly enhanced visual responses in fusiform and occipital cortex, extending to area V1. Literacy also enhanced phonological activation to speech in the planum temporale, and afforded a top-down activation of orthography from spoken inputs. Most changes occurred even when literacy was acquired in adulthood, emphasizing that both childhood and adult education can profoundly refine cortical organization.

Virtually all adult neuroimaging experiments are performed in highly educated college students. The observed brain architecture therefore reflects the influence of culture and education over and above spontaneous brain development (1, 2). Indeed, the acquisition of reading, a major event in children's lives, is now recognized as capable of changing both brain anatomy $(3,4)$ and brain activation $(5-9)$. In the auditory modality, literacy leads to phonemic awareness, the ability to manipulate the smallest units of spoken language, i.e. phonemes (10), and alters on-line speech processing (1114). At the visual level, developmental neuroimaging studies in normal and dyslexic children show that, with reading acquisition, a specific brain site in left occipito-temporal cortex, which has been termed "visual word form area" (VWFA), starts to respond to orthographic stimuli in the learned script (15-19).

These observations leave many important questions unanswered. First, does literacy primarily lead to cooperative or to competitive effects on cortical processing? Two theoretical positions can be contrasted. The first view, derived from animal studies of environmental enrichment and sensory plasticity, emphasizes that perceptual learning entails beneficial modifications of cortical maps, including sharpened receptive fields and neuronal tuning curves correlated with behavioral improvements (20-22). Without denying these positive effects, the second view emphasizes that reading is a cultural invention too recent to involve dedicated genetic or developmental mechanisms. Thus, during education, reading processes must invade and "recycle" cortical space devoted to evolutionary older functions, opening the possibility that these functions suffer as reading expertise sets in $(2,23)$. Much like expertise for non-face stimuli induces a reduction in face responses (24$26)$, reading, which recruits an identical cortical site in all cultures (27), might entail a reorganization of nearby responses to faces, houses and objects. We thus aimed to understand which of these stimuli are processed in the VWFA area prior to reading and how their cortical representation, which is known to increase during the school years (28), is affected by literacy.

A second issue is that, at present, most functional imaging studies of illiteracy only contrasted schooled versus unschooled adults. Because these studies did not include "exilliterate" adults who did not attend school but were alphabetized during adulthood, they confounded the effects of schooling and literacy. The only important exception (4) focused solely on how brain anatomy is changed by literacy. In the present study, we separated the functional effects of schooling and literacy by comparing illiterates, ex-illiterates, and adults schooled in childhood.

Populations studied and verification of literacy level. We scanned a total of 63 Portuguese and Brazilian participants. Our sample included 32 unschooled adults (10 illiterates and 22 ex-illiterates with variable reading skills), 
and 31 schooled and literate adults. The latter group included 11 literate subjects matched to the illiterates in socioeconomic status (SES) (29). Reading skills were verified through behavioral tasks of letter identification, word and pseudo-word reading (with or without speed pressure), and sentence reading (Fig. 1, fig. S1, and table S1). All tests revealed the same ordering of literacy, from Brazilian illiterates (ILB) to Portuguese ex-illiterates (EXP), Brazilian ex-illiterates (EXB), low-SES Brazilian literates (LB2), Portuguese literates (LP) and Brazilian literates (LB1). We therefore relied on whole-brain linear regressions with reading performance (number of stimuli read per minute) across all groups to identify the brain regions influenced by literacy. Once identified, each brain site was submitted to restricted comparisons of subgroups to evaluate the effects of schooling and literacy with maximal sensitivity (29).

We used three types of whole-brain fMRI runs: a "localizer" with horizontal and vertical checkerboards, written and spoken sentences, motor commands and calculation problems (fig. S2); three visual runs evaluating cortical responses to faces, houses, tools, letter strings, falsefonts, and moving checkerboards, while the participant focused on detecting a target star (fig. S3); and four auditory lexical-decision runs with spoken stimuli. To verify compliance, during the localizer, participants either heard or saw short verbal instructions to perform simple calculations or to click the left or right button. With spoken instructions, we observed classical regions for calculation and hand movements, without modulation by literacy, indicating similar comprehension and compliance in all groups. With written instructions, however, activation at the same locations was strongly modulated by reading performance, varying from zero activation in illiterates to a level equivalent to spoken instructions in literates (see fig. S4). While unsurprising, these results validate our group definitions and literacy measure, and establish that, with spoken materials, all groups followed instructions quite well. Thus, any subsequent differences cannot be attributed to lower attention or comprehension in illiterates.

Responses to written sentences enhanced by literacy. We first examined, in the localizer run, which regions were modulated by reading performance during the viewing of simple sentences consisting of serially presented written words (Fig. 2 and fig. S7). A massive effect was seen in the left ventral occipito-temporal cortex, at classical VWFA coordinates $(-40,-50,-14, Z=6.86)$, with posterior subpeaks ($46,-70,-18, Z=5.39 ;-32,-80,-8, Z=3.96)$ and a right occipital site (22, -86, $-10, Z=5.17$ ). These regions were strictly visual, as attested by their lack of activation to spoken sentences. Modulation by reading performance was also seen in a vast left-hemisphere language network which was also activated by spoken language in all groups: left posterior, middle and anterior superior temporal sulcus (STS; -50, -44, 6, Z=7.10; -54, $-12,-12, \mathrm{Z}=5.42$; ), left temporal pole ( -50 , $12,-24, Z=4.13)$, left and right premotor cortex $(-46,-2,52$, $Z=8.50 ; 46,4,40, Z=5.48)$, left inferior frontal gyrus $(-54,26$, $-6, Z=5.76)$, and left SMA $(-4,2,62, Z=6.33)$. A significant left-hemispheric asymmetry of this effect was observed in all areas except temporal pole and occipital cortex. Direct comparison of spoken versus written stimuli showed that, in the literate participants, frontal regions became equally activated by spoken versus written language, while temporal areas overlapped but still showed a significant difference favoring spoken language (fig. S8).

This analysis thus uncovered three simple effects: with the acquisition of literacy, written materials (1) activate right occipital cortex at the same level as checkerboards; (2) induce a strong activation in left ventral visual cortex, at the classical site of the visual word form area (VWFA); (3) gain access to left perisylvian temporal and frontal language areas.

The visual word form area: a major correlate of literacy. Our next analyses focused on visual responses in the VWFA. The effect of reading performance on occipitotemporal cortex during sentence reading was replicated when contrasting passive viewing of letter strings relative to rest (main peak $=-46,-80,4, Z=5.75$; subpeaks at $-40,-70,-12$, $\mathrm{Z}=4.50$; and the VWFA proper, $-46,-58,-10, \mathrm{Z}=4.11$; right occipital region, $24,-86,-10, Z=5.25$, corrected $\mathrm{p}<0.05$ by false detection rate analysis [FDR] (29)). In this part of the experiment, which involved viewing meaningless pseudowords during an easy target-detection task, only these visual regions were modulated by literacy, confirming their role in automatic orthographic coding (16). Importantly, the impact of schooling on the VWFA was replicated when comparing the illiterates with the matched low-SES literates (ILB $<$ LB2), both for written sentences versus rest (-40, -50, $14, \mathrm{Z}=6.77)$ and strings versus rest $(-48,-60,-10, \mathrm{Z}=3.54)$. The VWFA was also identified when searching for activation positively correlated with reading performance within the unschooled participants only (illiterates and ex-illiterates; sentences versus rest: $-42,-54,-6, Z=6.25$; strings versus rest: $-48,-56,-6, Z=3.53)$. This finding indicates that adult literacy suffices to establish it.

Literacy did not just amplify letter string responses, but also increased the cortical selectivity for this category relative to others. When testing the impact of reading performance on the difference between letter strings and other visual categories, only the VWFA appeared (peak at $-44,-56,-14$, $Z=5.00$; subpeak $-44,-68,-12, Z=3.85$ ). This effect showed a highly significant hemispheric asymmetry, peaking at the classical VWFA coordinates (-44, -56, -12, Z=5.07). Thus, literacy results in the emergence of a cortical site increasingly more responsive to writing than to other visual categories (17, 30). 
Group analyses left open the possibility of a selective but spatially variable response to written strings in every subject. Literacy would then merely displace this response to a reproducible site, without changing its amplitude. This possibility was refuted, however, through an individual analysis where the voxel most responsive to written sentences versus checkerboards was first identified in each participant, within $10 \mathrm{~mm}$ of the group peak (similar results were obtained with 20 or $40 \mathrm{~mm}$ ), and then analyzed for its responses to strings and falsefonts in the independent visual runs. We observed a significant lateral-to-mesial shift of the word-responsive peak (from $x=-48$ in illiterates to -46 in exilliterates and -44 in literates, $\mathrm{p}=0.006$ ), but its activation to strings also increased strongly with reading performance (linear regression, $\mathrm{r}^{2}=46.5 \%, \mathrm{p}<0.0001$ ), as did the selectivity index for strings relative to falsefonts $(\mathrm{p}=0.001)$. Thus literacy genuinely increases both the strength and specificity of cortical responses to the learned script in the VWFA.

We also searched for non-monotonic effects across our six groups, which might arise if the ex-illiterates had to mobilize a broader network than either literates or illiterates in order to read. Indeed, during sentence reading, Brazilian ex-illiterates showed greater activity than Brazilian literates in bilateral mesial fusiform/parahippocampal areas (-34, -60, 0, Z=5.34; $38,-50,-2, Z=4.54)$ and right posterior parietal cortex (24, 62, 38, Z=4.70; 12, -58, 60, Z=4.16) (fig. S5). Thus, to achieve their modest reading performance, ex-illiterates engage a broader and more bilateral ventral network than literates, and recruit additional posterior parietal regions associated with serial effortful reading (31). This observation is similar to the developmental finding that reading in young children initially involves a broad bilateral visual network (18) that progressively restricts to the VWFA as greater expertise sets in (19).

Competition with other visual categories in occipitotemporal cortex. At the peak coordinates of the VWFA (-44, $-50,-14$, identified by the localizer), analysis of the independent visual runs showed a strong response to strings but also to other visual categories, particularly faces and tools (Fig. 3). This finding confirms that this area plays a broad role in visual shape analysis. Reading being a recent invention, we expected that written words would not activate a fully dedicated cortical site, but would only partially "recycle" existing cortical mechanisms for visual recognition $(2,23)$, inducing a cortical competition that would increase with reading expertise. We tested the predicted cortical competition by searching for a decreasing response to visual stimuli with increasing reading performance. At the independently defined VWFA peak, the responses to checkerboards slightly diminished with reading performance, both across all groups (linear regression, $\mathrm{p}=0.013$ ), for illiterates compared to all other groups $(\mathrm{p}=0.025)$ and for illiterates compared to SES-matched literates (ILB $>$ LB2 comparison, $\mathrm{p}=0.039$ ). For houses and tools, only marginal decreasing trends were found (same three tests, $\mathrm{p}=0.08 / 0.025 / 0.09$ for houses and $0.13 / 0.025 / 0.06$ for tools). For faces, the decreasing tendency was stronger: the regression with reading performance across all participants was marginal ( $\mathrm{p}=0.09$ ), but the more focused comparisons were significant (ILB $>$ other groups, $p=0.025$; ILB $>$ LB2, $\mathrm{p}=0.003)$. Comparing illiterates with SES-matched literates indeed arguably provides a purer test, controlling for the possibility that frequency of exposure to faces might increase with socio-economic status and influence fusiform responses (32). When studied at the whole-brain level, the ILB $>$ LB2 contrast indicated a highly significant reduction of face response with literacy ( $\mathrm{p}<0.001$, cluster $\mathrm{p}<0.05$ corrected) in two bilateral posterior fusiform clusters (right: $40,-80,0$, $Z=5.93$, with an anterior subpeak, 38, $-50,-12, Z=4.70$; left, $44,-70,-12, Z=4.58$, with a subpeak precisely at the VWFA, $-42,-54,-14, Z=3.91)$. The same contrast did not reach corrected-level significance for houses or tools.

In summary, at the VWFA site, learning to read competes primarily with the cortical representation of checkers and faces. Further analyses showed that this competition was spatially restricted. We implemented analyses inspired by Golarai et al. (28), who showed that cortical peaks with adultlike selectivity to faces and places already exist in 7-11-yearsolds and, with increasing age, progressively expand into the surrounding cortex. For each subject, we first searched for the peak response to faces versus houses within $10 \mathrm{~mm}$ of the group coordinates of the VWFA. We then examined an orthogonal regression testing how the activation to faces varied with literacy, at the peak and in increasingly larger annuli of 2, 4, 6 or 8 voxels surrounding it (fig. S6). There was no change in peak face responses with reading performance ( $\mathrm{p}=0.47$ ), nor in annuli of radius 2 or 4 , but in the more distant annuli of radius 6 or 8 , face activation decreased with reading performance (regression across all groups, respectively $\mathrm{p}=0.037$ and $\mathrm{p}=0.015$ ). Similar findings were obtained for the individual peak of responsitivity to houses versus faces: no change in peak activation $(p=0.20)$, but a decrease in house-driven activation in the larger annulus of radius $8(\mathrm{p}=0.045)$. For tools, a category for which no selective region exists in ventral visual cortex (33), we did not find an annular reduction, but a more diffuse reduction in activation with reading performance, significant over both a large sphere of $16 \mathrm{~mm}(\mathrm{p}=0.039)$ and in the 50 voxels best responsive to tools versus houses $(\mathrm{p}=0.019)$, but again not at the peak itself $(\mathrm{p}=0.27)$.

Overall, our results indicate that the developmental competition induced by the expansion of orthographic representations in the ventral visual system is modest, does not directly affect the peak responses to faces and houses, but 
interferes with their expansion into the surrounding cortex. These conclusions fit with previous studies of visual development (28), expertise $(24,25)$, and plasticity of sensory maps (20), which reveal a displacement of map boundaries due to cortical competition.

Positive effects of literacy on visual organization. Competition could also have a positive effect on cortical responses to non-reading-related visual categories: by reducing the dispersion of their neural responses, it might force them to a more consistent cortical site. Indeed, a wholebrain search revealed positive correlations of reading performance with face and house responses in ventral occipito-temporal cortex (Fig. 3). Reading performance modulated positively the face versus rest contrast in the right anterior fusiform gyrus (38, -42, -20, Z=4.84, FDR), and induced a significant right-hemispheric shift of face responses in occipito-temporal cortex (24, -88, -10, $Z=8.02$; 36, -62, 12; Z=6.72; and 38, -40, -22, Z=4.77, FDR). Similarly, reading performance modulated the house versus rest contrast in bilateral mesial fusiform/parahippocampal regions (peaks at $34,-58,-12, Z=5.19 ; 24,-36,-16, Z=4.83$ ), with a righthemispheric asymmetry (36, $-60,-12, \mathrm{Z}=5.53)$. Altogether, these influences on word, face, and place responses resulted in a better differentiated mosaic of category-specific regions in ventral visual cortex in literates (Fig. 4). Importantly, however, face and house increases were found neither when comparing illiterates with their SES-matched literate group (ILB $<$ LB2 comparison), nor when testing for the effect of literacy in non-schooled participants only (table S2). Plots showed that these effects differentiated the participants living primarily in urban areas (LB1, LP and to a lesser extent EXP) versus those living in rural areas (ILB, EXB and LB2), regardless of their schooling and reading scores (Fig. 3). While these observations suggest an influence of familiarity rather than literacy or schooling per se, they are nevertheless important in showing how ventral fusiform organization can be affected by cultural variables (1).

Literacy led to another effect: a general enhancement of occipital responses. We probed the right occipital location identified as being modulated by reading performance during written sentences versus rest in the localizer run (coordinates $22,-86,-10)$. During the independent visual runs, the activation of this region to every visual category correlated tightly with reading scores, with the lowest correlation achieved with the checkerboards $\left(\mathrm{r}^{2}=0.08, \mathrm{p}=0.02\right.$; all other categories, $\mathrm{r}^{2}$ ranging from 0.17 to $0.22, \mathrm{p}<0.0007$ ).

Furthermore, these effects were genuinely related to literacy, not just schooling (table S2). When extended to a whole-brain search, using a main contrast for increasing activation to all visual categories, this effect was significant not only in right occipital (24, -84, -10, Z=14.6), but also left occipital cortex $(-48,-80,-4, Z=9.35)$ and a right occipito-parietal cluster (24,
-76, 36, Z=6.75), always with significant right-hemispheric asymmetry. Thus, literacy enhanced occipital responses to essentially all the contrasted black-and-white visual stimuli used in our study.

We also examined whether early retinotopic responses were affected. The localizer comprised horizontal and vertical checkerboards, designed to isolate the meridians of early visual maps. In the Roman alphabet, words appear as horizontally extended strings, and expert readers show enhanced behavioral processing of letter strings presented at the familiar foveal and horizontal location $(31,34)$. We therefore predicted that literacy might increase the responses to horizontal relative to vertical checkerboards. Indeed, this effect was observed at two symmetrical occipital sites corresponding to primary visual cortex $(16,-88,2 ; Z=5.10$; and $-12,-88,2 ; Z=4.75, F D R)$. These sites exhibited a strong response to horizontal, but not vertical checkerboards, and the modulation by reading performance was seen only with horizontal checkerboards (Fig. 3). Interestingly, this effect was significantly stronger in left than in right V1 (asymmetry effect for horizontal>vertical checkerboards, peak at -4, -88, $4, Z=5.37$, FDR). Both occipital sites also showed a positive correlation with literacy when written sentences were presented (table S2). Overall, these results suggest that literacy results in a form of perceptual learning (20-22) that refines the earliest stage of cortical visual processing. At this stage, learning is generic enough to generalize to checkerboard stimuli presented at the trained location. The greater effect in left area V1 fits with the larger letteridentification span in the right visual field in left-to-right readers (35).

Enhanced responses to spoken language. Finally, we examined how literacy affected spoken language processing (Fig. 5). Several regions showed a decreasing activation to spoken sentences with greater reading performance: left posterior STS (-44, $-52,18, Z=5.45)$, left and right middle temporal gyri (-66, $-22,-10, Z=5.25 ; 48,-30,-8, Z=5.34)$, and midline anterior cingulate cortex $(4,42,42, Z=4.11)$. These reductions probably reflect a facilitation of speech comprehension in literate participants (8). In the converse direction, however, activation to spoken sentences essentially doubled from illiterates to literates in left and right superior temporal regions just posterior to Heschl's gyrus (Planum temporale; -38, -28, 18, Z=5.52; 42, $-14,16, \mathrm{Z}=5.43$ ), with bilateral subpeaks near Heschl's gyrus (-60, $-14,10$; $Z=4.28$; 66, -2, 24, Z=4.41) and a significant left-hemispheric asymmetry. The effect was replicated in the independent auditory lexical decision runs, with both words and pseudowords (correlations with reading performance: $\mathrm{r}^{2}=0.20$, $\mathrm{p}=0.0002$; and $\left.\mathrm{r}^{2}=0.18, \mathrm{p}=0.0005\right)$. The enhanced temporal response was restricted to spoken language, with no trace of activation to written sentences at this site. 
The planum temporale is involved in phonological coding of speech (36) and is sensitive to the congruity between a speech sound and a simultaneous visually presented letter (37), an effect which is reduced or absent in dyslexic subjects (38). Our results make this region a prime candidate for the enhanced phonemic processing which accompanies alphabetization [(10-12), see also ref. (39)]. They also suggest that the reduced planum temporale activation seen in dyslexic children, rather than being a cause of dyslexia (38), could be a consequence of abnormal reading acquisition.

During auditory lexical decision, but not sentence listening, a second site in left fusiform cortex also increased its activation as a function of reading performance (-48, -52 , 8, Z=8.62). This site showed no activation in illiterates, but a strong one in all literate groups (Fig. 5). Its coordinates strongly suggest a top-down activation of the VWFA. Indeed, its activation to spoken words and pseudowords was positively correlated to its activation by written strings in the independent visual runs ( $r=+0.46$; no such correlation was found with other visual categories). Coincidence with the VWFA was also established by first identifying the peak response to written sentences versus checkerboards in each participant, and then correlating its activation during spoken lexical decision with the participant's reading performance $(p=0.005)$. Altogether, those results confirm that the VWFA can be activated in a top-down manner during speech processing (40-42), even in a lexical decision task that does not require orthographic processing. Because this activation is present only inasmuch as the participants can read, our findings suggest that it reflects the recruitment of an orthographic code rather than a generic picture code (43).

Previous psycholinguistic research has demonstrated that orthography affects spoken language processing (11-14), but it remains debated whether an orthographic code is activated on-line whenever we hear a spoken word, or whether orthography merely changes the nature of phonological representations (12). Our results show that both phenomena coexist: the planum temporale increase suggests enhanced phonological coding, compatible with a recent study using low-resolution electro-encephalography (12), while the VWFA activation indicates an additional and optional orthographic recruitment.

Effects of early schooling and late literacy. The above fMRI findings are based on global correlations of brain activation with reading performance, and therefore reflect the joint influences of schooling and literacy. To separate these variables, we performed additional regression analyses on all previously identified peaks (29). One analysis evaluated the impact of literacy acquired during adulthood by testing the effect of reading performance within unschooled subjects only. Another analysis, conversely, probed the impact of early schooling by regressing out the effect of reading performance and testing for a remaining difference between the Brazilian ex-illiterates (EXB) and the low-SES Brazilian literates (LB2 group), who only differed in early schooling.

The results were clear-cut. Virtually all of the above effects of literacy were present in ex-illiterates alphabetized during adulthood (table S2). Such was the case for the increased VWFA response to letter strings; the capacity to activate the spoken language network through reading (except in left temporal pole); the general visual increase in right occipital cortex; the greater response of area V1 to horizontal checkerboards and written sentences; and the enhanced planum temporale and top-down VWFA activation to spoken words and pseudowords. Thus, the neural modifications induced by adult literacy education were considerable. Furthermore, the vast majority were unaffected by early schooling (table S2). There were only two interesting exceptions. The first was the reduced activation to faces in the VWFA, which was indeed particularly prominent in the LB2 group, who benefitted from early schooling, relative to the EXB group. This finding suggests that competitive interactions between written words and faces in ventral visual cortex primarily occurs when reading is acquired in childhood, a time when visual maps are known to be highly malleable (28). The other effect of early schooling concerned a marginal left premotor increase in activation to written sentences. This region overlaps with Exner's writing center and is thought to code handwriting gestures (44). While earlyschooled participants were fluent in handwriting, it is possible that the ex-illiterates did not receive enough training to automatically activate a gesture code from the mere vision of written sentences.

As a cautionary note, we stress that these conclusions may only be valid for the moderate alphabetization courses experienced by our ex-illiterate participants. Whether earlyschooling effects truly reflect a limit on adult sensory plasticity, or would vanish with more intense reading practice, remains an open question. However, our results also indicate that, in literates, most of the observed effects do not change further as reading expertise increases (table S2; the only exceptions were increases in planum temporale and left pSTS, and reduced VWFA activation to checkerboards). In particular, the VWFA activation to words and strings increases briskly from illiterates to ex-illiterates and then reaches a plateau uncorrelated with ultimate proficiency, in agreement with developmental evidence that minimal literacy training suffices to establish it in 6-year-olds (18).

Conclusion. Literacy, whether acquired in childhood or through adult classes, enhances brain responses in at least three distinct ways. First, it boosts the organization of visual cortices, particularly by inducing an enhanced response to the known script at the VWFA site in left occipito-temporal cortex and by augmenting early visual responses in occipital 
cortex, in a partially retinotopic manner. Second, literacy allows virtually the entire left-hemispheric spoken language network to be activated by written sentences. Thus reading, a late cultural invention, approaches the efficiency of the human species' most evolved communication channel, namely speech. Third, literacy refines spoken language processing by enhancing a phonological region, the planum temporale, and by making an orthographic code available in a top-down manner. These largely positive changes should not hide that literacy, like other forms of expertise, also leads to cortical competition effects (23-26). At the VWFA site, a significantly reduced activation was found for checkerboards and faces. The intriguing possibility that our face perception abilities suffer in proportion to our reading skills will be explored in future research.

\section{References and Notes}

\section{J. Henrich, S. J. Heine, A. Norenzayan, Behav Brain Sci}

33, 61 (2010).

2. S. Dehaene, L. Cohen, Neuron 56, 384 (2007).

3. A. Castro-Caldas et al., Eur J Neurol 6, 23 (1999).

4. M. Carreiras et al., Nature 461, 983 (2009).

5. K. M. Petersson, C. Silva, A. Castro-Caldas, M. Ingvar, A. Reis, Eur J Neurosci 26, 791 (2007).

6. G. Li et al., Hum Brain Mapp 27, 144 (2006).

7. K. M. Petersson, A. Reis, S. Askelof, A. Castro-Caldas, M. Ingvar, J Cogn Neurosci 12, 364 (2000).

8. A. Castro-Caldas, K. M. Petersson, A. Reis, S. StoneElander, M. Ingvar, Brain 121, 1053 (1998).

9. K. M. Petersson, A. Reis, A. Castro-Caldas, M. Ingvar, Neuroimage 10, 45 (1999).

10 J. Morais, P. Bertelson, L. Cary, J. Alegria, Cognition 24, 45 (1986).

11. H. Cheung, H. C. Chen, Language and Cognitive Processes 19, 1 (2004).

12. L. Perre, C. Pattamadilok, M. Montant, J. C. Ziegler, Brain Res 1275, 73 (2009).

13. P. Ventura, J. Morais, R. Kolinsky, Cognition 105, 547 (2007).

14. J. C. Ziegler, A. Petrova, L. Ferrand, J Exp Psychol Learn Mem Cogn 34, 643 (2008).

15. B. A. Shaywitz et al., Biol Psychiatry 52, 101 (2002).

16. L. Cohen, S. Dehaene, Neuroimage 22, 466 (2004).

17. C. I. Baker et al., Proc Natl Acad Sci U S A 104, 9087 (2007).

18. S. Brem et al., Proc Natl Acad Sci U S A 107, 7939 (2010).

19. U. Maurer et al., Neuroimage 33, 749 (2006).

20. D. B. Polley, E. E. Steinberg, M. M. Merzenich, $J$ Neurosci 26, 4970 (2006).

21. W. Li, V. Piech, C. D. Gilbert, Nat Neurosci 7, 651 (2004).

22. M. Sigman et al., Neuron 46, 823 (2005).
23. S. Dehaene, Reading in the Brain (Penguin Viking, New York, 2009).

24. I. Gauthier, T. Curran, K. M. Curby, D. Collins, Nat Neurosci 6, 428 (2003).

25. A. Harel, S. Gilaie-Dotan, R. Malach, S. Bentin, Cereb Cortex 20, 2304 (2010).

26. B. Rossion, C. C. Kung, M. J. Tarr, Proc Natl Acad Sci U $S$ A 101, 14521 (2004).

27. D. J. Bolger, C. A. Perfetti, W. Schneider, Hum Brain Mapp 25, 92 (2005).

28. G. Golarai et al., Nat Neurosci 10, 512 (2007).

29. Materials and methods are available as supporting material on Science Online.

30. R. Gaillard et al., Neuron 50, 191 (2006).

31. L. Cohen, S. Dehaene, F. Vinckier, A. Jobert, A. Montavont, Neuroimage 40, 353 (2008).

32. E. Eger, S. R. Schweinberger, R. J. Dolan, R. N. Henson, Neuroimage 26, 1128 (2005).

33. P. E. Downing, A. W. Chan, M. V. Peelen, C. M. Dodds, N. Kanwisher, Cereb Cortex 16, 1453 (2005).

34. T. A. Nazir, N. Ben-Boutayab, N. Decoppet, A. Deutsch, R. Frost, Brain Lang 88, 294 (2004).

35. K. Rayner, Psychol Bull 124, 372 (1998).

36. C. Jacquemot, C. Pallier, D. LeBihan, S. Dehaene, E. Dupoux, J Neurosci 23, 9541 (2003).

37. N. van Atteveldt, E. Formisano, R. Goebel, L. Blomert, Neuron 43, 271 (2004).

38. V. Blau et al., Brain 133, 868 (2010).

39. P. E. Turkeltaub, L. Gareau, D. L. Flowers, T. A. Zeffiro, G. F. Eden, Nat Neurosci 6, 767 (2003).

40. Y. N. Yoncheva, J. D. Zevin, U. Maurer, B. D. McCandliss, Cereb Cortex 20, 622 (2010).

41. A. S. Desroches et al., Brain Res 1356, 73 (2010).

42. J. R. Booth et al., Hum Brain Mapp 19, 155 (2003).

43. F. Kherif, G. Josse, C. J. Price, Cereb Cortex, 22 April 2010 (10.1093/cercor/bhq063).

44. M. Longcamp, J. L. Anton, M. Roth, J. L. Velay, Neuroimage 19, 1492 (2003).

45. This work was supported by Institut National de la Santé et de la Recherche Médicale (INSERM), Commissariat à l'Energie Atomique (CEA), Collège de France, Agence Nationale de la Recherche (project CORELEX), Fundação para a Ciência e a Tecnologia, the European Community FEDER funding (Project PTDC/PSI/66077/2006, “Cognitive consequences of literacy”), SARAH Network of Rehabilitation Hospitals, and by two grants from the Belgian French community (FRFC 2.4586.07 and ARC 06/11-342). We gratefully acknowledge Alexis Amadon, Marie-Hélène Baju, Laurence Labruna, Denis Le Bihan, Lucie Hertz-Pannier, Philippe Pinel and the NeuroSpin infrastructure groups at NeuroSpin, and Denis Yudi Suguieda, Renata Santinoni Vera, Alexandre Guerreiro 
Tauil, Eduardo Amemiya, Luiz Guilherme Nadal Nunes, Sandro Barbosa de Oliveira and Cynthia L. M. Gillis at SARAH Brasilia.

\section{Supporting Online Material}

www.sciencemag.org/cgi/content/full/science.1194140/DC1

Materials and Methods

SOM Text

Figs. S1 to S8

Tables S1 and S2

References

23 June 2010; accepted 20 October 2010

Published online 11 November 2010;

10.1126/science. 1194140

Fig. 1. The six groups of participants and their reading skills. Box plots show the speed and accuracy in reading a list of pseudowords (central mark $=$ median, box $=25^{\text {th }}$ and $75^{\text {th }}$ percentiles, whiskers $=\min$ and $\max$ ). Additional data on word and sentence reading are provided in fig. S1.

Fig. 2. Effects of literacy on brain responses to written sentences during the localizer. Axial and sagittal slices show voxels where activation was modulated by literacy during exposure to written sentences relative to rest (voxel $\mathrm{p}<0.001$, cluster $\mathrm{p}<0.05$ corrected). Colored labels refer to participant groups and are ordered according to reading performance (see Fig. 1). Plots report activation to visual checkers, spoken and written language relative to rest in the localizer run, in arbitrary units (mean \pm one standard error). To avoid circularity, plots were generated solely from data independent from the voxel-selection criterion.

Fig. 3. Effects of literacy on visual responses to different categories of stimuli. (A) Visual responses in the VWFA. Inset = SPM map of modulation by literacy of activation to written sentences in the localizer, $\mathrm{p}<0.001$, cluster corrected $\mathrm{p}<0.05$ ). Plot $=$ activation in the independent visual runs at the isolated peak. Activation increased in response to letter strings ( $p=0.005)$ and decreased for checkerboards $(p=0.013)$ and, to a lesser extent, for faces (overall $p=0.09$, ILL $>$ LB2, $\mathrm{p}=0.003$ ). ( $\mathbf{B}$ and $\mathbf{C}$ ) SPM maps (voxel $\mathrm{p}<0.001$ uncorrected) and peak plots showing increases with reading performance of face responses in right anterior fusiform, and of house responses in bilateral parahippocampal regions. (D) SPM map of enhancement by literacy of the response to written sentences in lateral occipital cortex (inset, $\mathrm{p}<0.001$, cluster corrected $\mathrm{p}<0.05$ ), and plot showing its replication in response to other visual categories. (E) Modulation by literacy of the greater response to horizontal than to vertical checkerboards in primary visual cortex (inset, voxel $\mathrm{p}<0.001$ uncorrected). In panels (B), (C), and (E), plots are provided for illustration, as they do no arise from independent data; plots (A) and (D) are from independent data.

Fig. 4. Mosaic of preferences for different visual categories in ventral visual cortex. Slices at right show the activation difference between a given category and all the others (for greater comparability between groups with different numbers of subjects, the figure does not show statistical t maps, but BOLD signal maps arbitrarily thresholded at $0.66 \%$ of the mean BOLD signal over the whole brain; similar results were seen with t maps). Graphs at left shows the evolution of the signal relative to rest for the different categories, at successive cortical sites tracing a horizontal line through the classical coordinates of the VWFA (-42, -57, -12; dotted line).

Fig. 5. Effects of literacy on brain responses to spoken language. (A) SPM maps of the effect of literacy on the activation to spoken sentences (left), words and pseudowords (right; voxel $\mathrm{p}<0.001$, cluster-size corrected $\mathrm{p}<0.05$ ). Plots show the effect observed in the left planum temporale: activation to spoken language, words and pseudowords was doubled or more after the acquisition of literacy (peak selected using the localizer run, left panel, and replicated in the independent lexical decision runs, right panel). (B) SPM maps of the effect of literacy on activations in the VWFA to spoken language. Literacy modulated activation to words and pseudowords during auditory lexical decision (right), but not during mere listening to spoken commands. Plots show the response at the a-priori location of the VWFA, as defined by the effect of literacy on written sentences (same location as in Figs. 2 and 3A). 


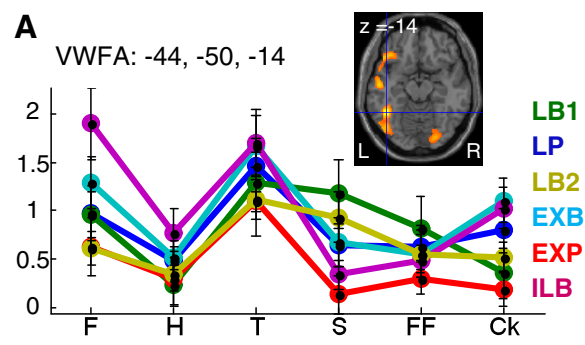

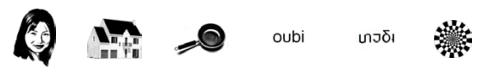

B

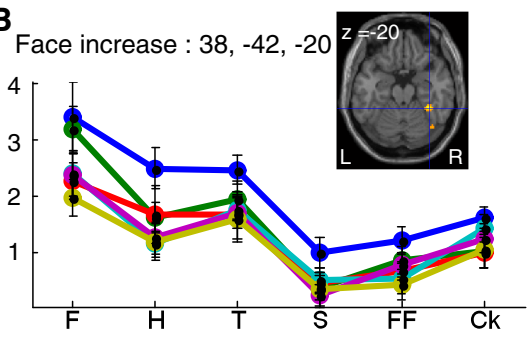

C

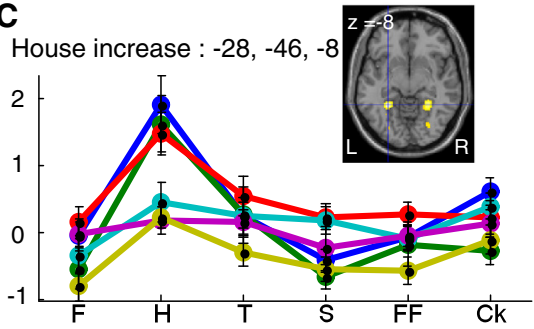

D

Global increase : $24,-84,-10$

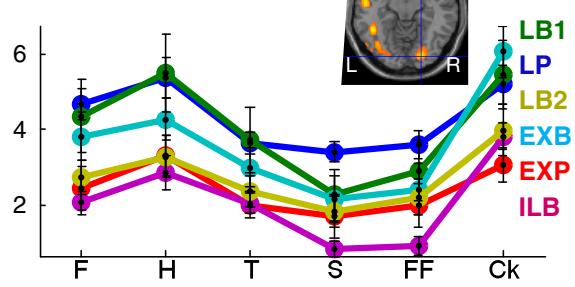

E Increased response to horizontal checkers

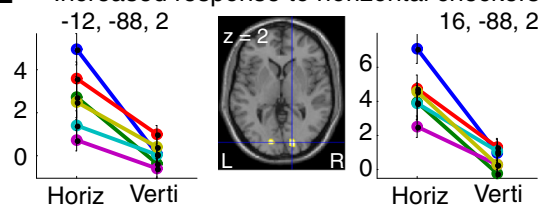




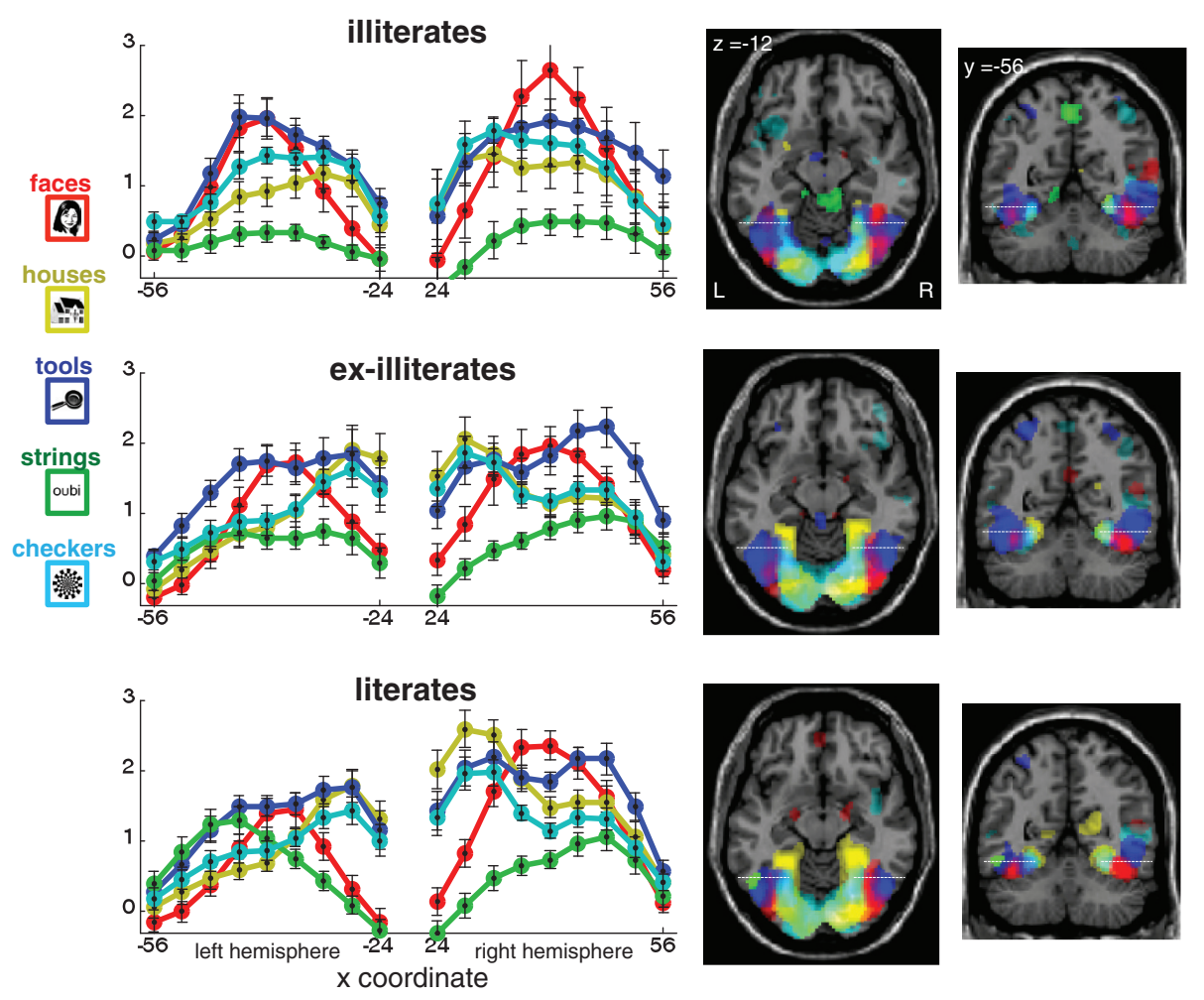


Spoken sentences in localizer

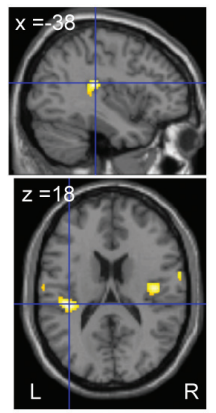

Response at [-38, -28, 18]

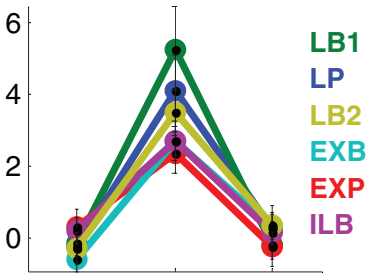

Checkers Spoken Written commands
Auditory lexical decision

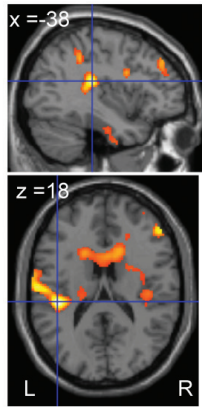

Response at $[-38,-28,18]$

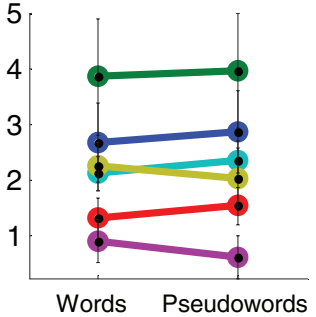

B Top-down activation of VWFA during auditory lexical decision

Spoken sentences in localizer

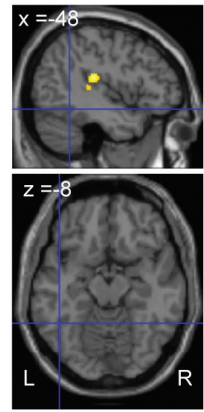

Response at [-44, -50, -14]

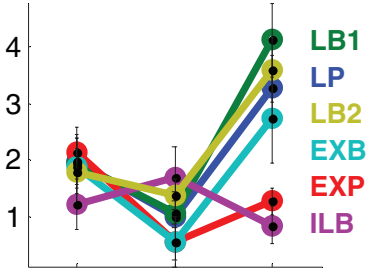

Checkers Spoken Written commands
Auditory lexical decision

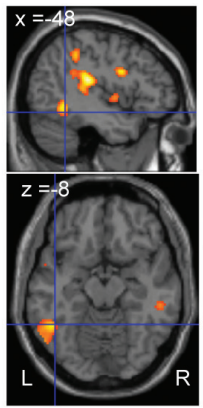

Response at [-44, -50, -14]

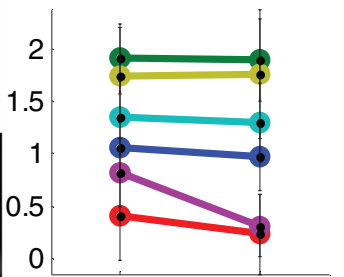

Words Pseudowords 


\section{Science}

\section{How Learning to Read Changes the Cortical Networks for Vision and Language}

Stanislas Dehaene, Felipe Pegado, Lucia W. Braga, Paulo Ventura, Gilberto Nunes Filho, Antoinette Jobert, Ghislaine Dehaene-Lambertz, Régine Kolinsky, José Morais and Laurent Cohen

published online November 11, 2010

ARTICLE TOOLS

SUPPLEMENTARY

MATERIALS

PERMISSIONS http://science.sciencemag.org/content/early/2010/11/10/science.1194140

http://science.sciencemag.org/content/suppl/2010/11/08/science.1194140.DC1 http://science.sciencemag.org/content/suppl/2010/11/11/science.1194140.DC2

http://www.sciencemag.org/help/reprints-and-permissions

Use of this article is subject to the Terms of Service

Science (print ISSN 0036-8075; online ISSN 1095-9203) is published by the American Association for the Advancement of Science, 1200 New York Avenue NW, Washington, DC 20005. The title Science is a registered trademark of AAAS.

Copyright @ 2010, American Association for the Advancement of Science 